\title{
On Distribution of Order Statistics from Kumaraswamy Dis- tribution
}

\author{
Mridula GarG \\ Department of Mathematics, University of Rajasthan, Jaipur-302004, India \\ e-mail : gargmridula@gmail.com
}

\begin{abstract}
In the present paper we derive the distribution of single order statistics, joint distribution of two order statistics and the distribution of product and quotient of two order statistics when the independent random variables are from continuous Kumaraswamy distribution. In particular the distribution of product and quotient of extreme order statistics and consecutive order statistics have also been obtained. The method used is based on Mellin transform and its inverse.
\end{abstract}

\section{Introduction}

If the random variables $X_{1}, X_{2}, \cdots X_{n}$ are arranged in ascending order of magnitudes and then written as $X_{(1)} \leq X_{(2)} \leq \cdots \leq X_{(n)}$

Then $X_{(i)}$ is called the $i^{t} h$ order statistics $i=1, \cdots, n$. The unordered random variables $X_{i}$ are usually statistically independent and identically distributed but the ordered random variables $X_{(i)}, i=1,2, \cdots, n$ are necessarily dependent. The distribution of product and quotient of random variables finds an important place in the literature and much work is done when the random variables are independent. However Subramaniam [9] has derived the distribution of the product and quotient of order statistics from a uniform distribution and negative exponential distribution respectively. Further Trudel and Malik [6] have derived the distribution of product and ratio of order statistics from Pareto, power and Weibull distributions.

Order statistics play an important supporting role in the multiple comparisons and multiple decision procedures such as the distribution of extreme order statistics $X_{(1)}, X_{(n)}$ and that of consecutive order statistics $X_{(i)}, X_{(i+1)}$ are often found useful in ranking [3] and selection [4] problems.

In the present paper we shall derive the distribution of product and quotient of order statistics from a Kumaraswamy distribution [11]. In probability theory Kumarswamy's double bounded distribution is as versatile as the Beta distribution, but much simpler to use especially in simulation studies as it has a simple closed form for both the probability density function and cumulative distribution function.

Received February 16, 2007.

2000 Mathematics Subject Classification: 60E99, 33C60.

Key words and phrases: Fox H function, Kumaraswamy distribution, Mellin transform, order statistics, random variable. 
The probability density function of a random variable $\mathrm{X}$ is given by

$$
f_{X}(x)=\left\{\begin{array}{lll}
a b x^{a-1}\left(1-x^{a}\right)^{b-1} & ; & 0 \leq x \leq 1 \\
0 & ; & \text { otherwise }
\end{array}\right.
$$

where $\mathrm{a}>0, \mathrm{~b}>0$.

The cumulative density function (or distribution function) is

$$
F_{X}(x)=1-\left(1-x^{a}\right)^{b}
$$

In particular if $\mathrm{a}=\mathrm{b}=1$, it gives the well known uniform distribution [10].

We shall require the following definition of Fox H-function [7]

$$
H_{p, q}^{m, n}\left[x \mid \begin{array}{c}
\left(a_{j}, \alpha_{j}\right)_{1, p} \\
\left(b_{j}, \beta_{j}\right)_{1, q}
\end{array}\right]=\frac{1}{2 \pi \omega} \int_{L} \theta(s) x^{s} d s,
$$

where $\omega=\sqrt{(-1)} x(\neq 0)$ is a complex variable and $x^{s}=\exp [s\{\log |x|+\omega \arg x\}]$

$$
\theta(s)=\frac{\prod_{j=1}^{m} \Gamma\left(b_{j}-\beta_{j} s\right) \prod_{j=1}^{n} \Gamma\left(1-a_{j}+\alpha_{j} s\right)}{\prod_{j=m+1}^{q} \Gamma\left(1-b_{j}+\beta_{j} s\right) \prod_{j=n+1}^{p} \Gamma\left(a_{j}-\alpha_{j} s\right)}
$$

$\mathrm{m}, \mathrm{n}, \mathrm{p}$, and $\mathrm{q}$ are non-negative integers satisfying $0 \leq n \leq p, 1 \leq m \leq q$ ; $\alpha_{j}(j=1, \cdots, p)$ and $\beta_{j}(j=1, \cdots, q)$ are assumed to be positive quantities for standardization purposes.

The definition of the $\mathrm{H}$-function given by (3) will however have meaning even if some of these quantities are zero, giving us in turn simple transformation formulas. The nature of contour L, a set of sufficient conditions for the convergence of this integral, the asymptotic expansion, some of its properties and special cases can be referred to the book by Srivastava, Gupta, Goyal [7].

\section{The distribution of single order statistics}

Theorem 1. Let $X_{(1)}, \cdots, X_{(n)}$ denote the order statistics of a random sample $X_{1}, \cdots, X_{n}$ of size $n$ from a continuous population with the probability density function and cumulative density function given by (1) and (2) respectively, then the p.d.f. of $j^{\text {th }}$ order statistics $X_{(j)}$ is given by

(5) $\quad f_{X_{(j)}}(x)=\frac{n ! a b^{j} x^{a j-1}}{(j-1) !(n-j) !}\left(1-x^{a}\right)^{b(n-j+1)-1}\left\{{ }_{2} F_{1}\left(1-b, 1,2, x^{a}\right)\right\}^{j-1}$,

$0 \leq x \leq 1,1 \leq j \leq n, a>0, b>0$

Proof. The formula for p.d.f. of jth order statistics $X_{(j)}$ is given by [8, p.28 eq.(3.5)]

$$
f_{X(j)}(x)=\frac{n !}{(j-1) !(n-j) !} f_{X}(x)\left[F_{X}(x)\right]^{j-1}\left[1-F_{X}(x)\right]^{(n-j)},
$$


where $f_{X}(x)$ and $F_{X}(x)$ denote the p.d.f. and c.d.f. of random variable $X$.

Substituting the values of $f_{X}(x)$ and $F_{X}(x)$ from (1) and (2) and simplifying the terms using some series manipulations we get the result in terms of Gauss hypergeometric function as given by (5).

\section{Special Cases.}

1. If we take $j=n$, we get p.d.f. of maximum of $X_{(j)}$ (say V) as

$$
f_{V}(v)=n a b^{n} v^{a n-1}\left(1-v^{a}\right)^{b-1}\left\{{ }_{2} F_{1}\left[1-b, 1,2, v^{a}\right]\right\}^{n-1} .
$$

2. On taking $j=1$, we get the p.d.f. of minimumof $X_{(j)}$ (say u) as

$$
f_{U}(u)=n a b u^{a-1}\left(1-u^{a}\right)^{b n-1} .
$$

3. If we take $a=b=1$, then we get p.d.f. of $j^{\text {th }}$ uniform order statistics as

$$
f_{X_{(j)}}(x)=\frac{n ! x^{j-1}}{(j-1) !(n-j) !}(1-x)^{(n-j)} .
$$

\section{Joint distribution of two order statistics}

Theorem 2. The joint p.d.f. of two order statistics $X_{(i)}$ and $X_{(j)}, 1 \leq i<j \leq n$ is given by

$$
\begin{aligned}
& f_{X_{(i)}, X_{(j)}}\left(x_{i}, x_{j}\right)=g\left(x_{i}, x_{j}\right) \\
= & \frac{n !(a b)^{2} x_{i}^{a-1} x_{j}^{a-1}}{(i-1) !(j-i-1) !(n-j) !}\left(1-x_{i}^{a}\right)^{b(j-1)-1}\left(1-x_{j}^{a}\right)^{b(n-j+1)-1} \\
& \times{ }_{1} F_{0}\left[1-i ;-;\left(1-x_{i}^{a}\right)^{b}\right]{ }_{1} F_{0}\left[1-j+i ;-;\left(\frac{1-x_{j}^{a}}{1-x_{i}^{a}}\right)^{b}\right],
\end{aligned}
$$

$a>0, b>0,0 \leq x_{(i)}<x_{(j)} \leq 1$.

Proof. The joint p.d.f. of two order statistics $X_{(i)}$ and $X_{(j)}$ is $(i<j)$ is given by [5, p.10]

$$
\begin{aligned}
& f_{X_{(j)}, X_{(j)}}\left(x_{i}, x_{j}\right)=\frac{n !}{(i-1) !(j-i-1) !(n-j) !} \\
& \quad \times\left[F\left(x_{i}\right)\right]^{i-1}\left[F\left(x_{j}\right)-F\left(x_{i}\right)\right]^{(j-i-1)}\left[1-F\left(x_{j}\right)\right]^{(n-j)} f\left(x_{i}\right) f\left(x_{j}\right)
\end{aligned}
$$

Substituting the values from (1) and (2) and making some simplifications we easily arrive at (10). 


\section{Special Cases.}

1. If we take $j=i+1$ in (10), we get the joint p.d.f. of two consecutive order statistics

$$
\begin{aligned}
g\left(x_{i}, x_{i+1}\right)= & \frac{n !(a b)^{2} x_{i}^{a-1} x_{i+1}^{a-1}}{(i-1) !(n-i-1) !}\left(1-x_{i}^{a}\right)^{b-1}\left(1-x_{i+1}^{a}\right)^{b(n-i)-1} \\
& \times{ }_{1} F_{0}\left[1-i ;-;\left(1-x_{i}^{a}\right)^{b}\right] .
\end{aligned}
$$

2. For $i=1$ and $j=n$, we obtain the joint p.d.f. of extreme order statistics as follows

$$
\begin{aligned}
g(u, v)= & n(n-1)(a b)^{2} u^{a-1} v^{a-1}\left(1-u^{a}\right)^{b(n-1)-1}\left(1-v^{a}\right)^{b-1} \\
& \times \exp \left\{\left(1-u^{a}\right)^{b}\right\}\left[1-\left(\frac{1-u^{a}}{1-v^{a}}\right)^{b}\right]^{n-2}, 0 \leq u<v \leq 1 .
\end{aligned}
$$

3. On taking $\mathrm{a}=\mathrm{b}=1$, we get the joint p.d.f. of two uniform order statistics after a little simplification [10, p.348, eq.(4.8.6)]

$$
g\left(x_{i}, x_{j}\right)=\frac{n ! x_{i}^{i-1}}{(i-1) !(j-i-1) !(n-j) !}\left(1-x_{j}\right)^{n-j}\left(x_{j}-x_{i}\right)^{j-i-1} .
$$

\section{The distribution of product and quotient of two order statistics}

Theorem 3. Let $X_{(i)}, X_{(j)}$ denote the $i^{\text {th }}$ and $j^{\text {th }}$ order statistics from a random sample of size $n$ drawn from Kumaraswamy distribution defined by (1). Then the probability density function $h(y)$ of product of order statistics $X_{(i)}$, and $X_{(j)}$ i.e.,

$$
Y=X_{(i)} X_{(j)} \quad(1 \leq i<j \leq n)
$$

is given by

(16) $h(y)=\frac{n ! a b^{2} y^{a-1}}{(i-1) !(j-i-1) !(n-j) !}\left(1-y^{a}\right)^{b(n-i+1)-1}$

$$
\begin{aligned}
& \times \sum_{m, l=0}^{\infty} \frac{(1-i)_{l}(1+i-j)_{m}}{l ! m !} \frac{\Gamma[b(j-i+l-m)] \Gamma[b(n-j+m+1)]}{\Gamma[b(l+n-i+1)]}\left(1-y^{a}\right)^{b l} \\
& \times{ }_{2} F_{1}\left[b(n-j+m+1), b(j+l-i-m) ; b(l+n-i+1) ; 1-y^{a}\right]
\end{aligned}
$$

provided that $j-i+l-m>0, a>0, b>0$.

Proof. The double Mellin transform [2] of the p.d.f. $\mathrm{h}(\mathrm{y})$ is given by $[10, \mathrm{p} .153$, eq.(4.8.6)]

$$
M_{s_{1}, s_{2}}[h(y)]=M_{s, s}[h(y)]=\int_{0}^{\infty} \int_{0}^{\infty} x_{i}^{s-1} x_{j}^{s-1} g\left(x_{i}, x_{j}\right) d x_{i} d x_{j},
$$


where $\mathrm{g}\left(x_{i}, x_{j}\right)$ denotes the joint p.d.f. of order statistics $X_{(i)}$ and $X_{(j)}$ and is given by (10). Substituting the values in (17), expressing hypergeometric function in their series form and evaluating the integrals with the help of a known result [1, p.311, eq. (31)], we get

$$
\text { (18) } \begin{aligned}
M_{s, s}[h(y)]=\frac{n ! a b^{2} y^{a-1}}{(i-1) !(j-i-1) !(n-j) !} \sum_{m, l=0}^{\infty}\left\{\frac{(1-i)_{l}(1+i-j)_{m}}{l ! m !}\right. \\
\left.\times \frac{\Gamma[b(j-i+l-m)] \Gamma[b(n-j+m+1)]}{\Gamma\left[1+b(l-m-i+j)+\frac{s}{a}-\frac{1}{a}\right]} \frac{\Gamma\left[1+\frac{s}{a}-\frac{1}{a}\right] \Gamma\left[1+\frac{s}{a}-\frac{1}{a}\right]}{\Gamma\left[1+b(n-j+m+1)+\frac{s}{a}-\frac{1}{a}\right]}\right\}
\end{aligned}
$$

On taking inverse Mellin transform [2] of (18) w.r.t. s, substituting s/a $=\mathrm{S}$ and using the result given in $[10$, p. 115], we arrive at the desired result (16).

\section{Special Cases.}

1. If we take $i=1, j=n$ in (16), we find that the series over 1 gets eliminated and the condition $j-i+l-m>0$ gives $\mathrm{m}<n-1$. On further making some simplifications we get the following result

$$
\begin{aligned}
& h_{1}(y)=n(n-1) a b^{2} y^{a-1}\left(1-y^{a}\right)^{b n-1} \sum_{m=0}^{n-2} \frac{(2-n)_{m}}{m !} \Gamma[b(n-m-1)] \\
& \quad \times \Gamma[b(m+1)]_{2} F_{1}\left[b(m+1), b(n-m-1) ; b n ;\left(1-y^{a}\right)\right],
\end{aligned}
$$

where $Y=U V \quad 0 \leq y \leq 1$.

2. If we take $j=i+1$, in (16), the summation over m gets removed and we get the distribution of product of two consecutive order statistics as

$$
\begin{aligned}
& h_{2}(y)=\frac{n ! a b^{2} \Gamma[b(n-i)]}{(i-1) !(n-i-1) !} y^{a-1}\left(1-y^{a}\right)^{b(n-i+1)-1} \\
& \times \sum_{l=0}^{\infty} \frac{(1-i)_{l}}{l !} \frac{\Gamma[b(l+1)] \Gamma[b(n-i)]}{\Gamma[b(l+n-i+1)]}\left(1-y^{a}\right)^{b l} \\
& \times{ }_{2} F_{1}\left[b(n-i), b(l+1) ; b(l+n-i+1) ;\left(1-y^{a}\right)\right],
\end{aligned}
$$

where $Y=X_{(i)} X_{(i+1)} \quad 0 \leq y \leq 1$.

3. In the above result if we further take $a=b=1$, we get a known result [10, p.349, eq.(9.7.6)]

Theorem 4. Let $X_{(i)}$ and $X_{(j)}$ be the $i^{\text {th }}$ and $j^{\text {th }}$ order statistics $(i<j)$ from a random sample of size $n$ drawn from the Kumaraswamy distribution defined by (1). Then the probability density function $h(z)$ of ratio

$$
Z=X_{(i)} / X_{(j)}(1 \leq i<j \leq n)
$$


is given by

$$
\begin{aligned}
(22) & h(z)=\frac{n ! b^{2}}{(i-1) !(j-i-1) !(n-j) !} \sum_{m, l=0}^{\infty} \frac{(1-i)_{l}(1+i-j)_{m}}{l ! m !} \Gamma[b(j-i+l-m)] \\
& \times \Gamma[b(n-j+m+1)] \mathrm{H}_{2,2}^{1,1}\left[\frac{1}{z} \mid \begin{array}{c}
\left(\frac{1}{a}, \frac{1}{a}\right),\left(1+b(n-j+m+1)+\frac{1}{a}, \frac{1}{a}\right) \\
\left(1+\frac{1}{a}, \frac{1}{a}\right),\left(b(i-j+m-l)+\frac{1}{a}, \frac{1}{a}\right)
\end{array}\right]
\end{aligned}
$$

provided that $j-i+l-m>0, a>0, b>0$. Here $H[x]$ denotes Fox H-function defined by (3).

Proof. The double Mellin transform of the p.d.f. $\mathrm{h}(\mathrm{z})$ is given by [10, p.153, eq. $(4.8 .7)]$

$$
M_{s_{1}, s_{2}}[h(z)]=M_{s, 2-s}[h(z)]=\int_{0}^{\infty} \int_{0}^{\infty} x_{i}^{s-1} x_{j}^{(2-s)-1} g\left(x_{i}, x_{j}\right) d x_{i} d x_{j} .
$$

Substituting the value of joint density function $\mathrm{g}\left(x_{i}, x_{j}\right)$ from eq.(10), expressing both ${ }_{1} F_{0}$ in series form and using the result [1, p.311, eq.(31)], we get

$$
\begin{aligned}
& M_{s, 2-s}[h(z)]=\frac{n ! b^{2}}{(i-1) !(j-i-1) !(n-j) !} \sum_{m, l=0}^{\infty}\left\{\frac{(1-i)_{l}(1+i-j)_{m}}{l ! m !}\right. \\
& \left.\times \frac{\Gamma[b(j-i+l-m)] \Gamma[b(n-j+m+1)]}{\Gamma\left[1+b(l-m-i+j)-\frac{1}{a}+\frac{s}{a}\right]} \frac{\Gamma\left[1+\frac{s}{a}-\frac{1}{a}\right] \Gamma\left[1-\frac{s}{a}+\frac{1}{a}\right]}{\Gamma\left[1+b(n-j+m+1)+\frac{1}{a}-\frac{s}{a}\right]}\right\}
\end{aligned}
$$

On taking inverse Mellin transform of (24) with respect to s and using the definition (3), we arrive at (22).

\section{Special Cases.}

1. If we take $i=1, j=n$ in (22), the summation over $l$ is eliminated and we obtain the distribution of quotient of extreme order statistics

$$
\begin{aligned}
h_{1}(z)=\frac{n ! b^{2}}{(n-2) !} \sum_{m=0}^{n-2} \frac{(2-n)_{m}}{m !} \Gamma[b(n-m-1)] \Gamma[b(m+1)] \\
\times \mathrm{H}_{2,2}^{1,1}\left[\begin{array}{c|c}
\frac{1}{z} & \left(\frac{1}{a}, \frac{1}{a}\right),\left(1+b(m+1)+\frac{1}{a}, \frac{1}{a}\right) \\
\left(1+\frac{1}{a}, \frac{1}{a}\right),\left(b(1+m-n)+\frac{1}{a}, \frac{1}{a}\right)
\end{array}\right],
\end{aligned}
$$

where $Z=U / V$.

2. If we take $j=i+1$ in (22), $\mathrm{m}$ takes value zero and we obtain the distribution 
of quotient of consecutive order statistics

$$
\begin{aligned}
& \text { (26) } h_{2}(z)=\frac{n ! b^{2}}{(i-1) !(n-i-1) !} \sum_{l=0}^{\infty} \frac{(1-i)_{l}}{l !} \Gamma[b(l+1)] \Gamma[b(n-i+m+2)] \\
& \times \mathrm{H}_{2,2}^{1,1}\left[\begin{array}{c|c}
\left(\frac{1}{a}, \frac{1}{a}\right),\left(1+b(n-i)+\frac{1}{a}, \frac{1}{a}\right) \\
\left(1+\frac{1}{a}, \frac{1}{a}\right),\left(-b(l+1)+\frac{1}{a}, \frac{1}{a}\right)
\end{array}\right],
\end{aligned}
$$

where $Z=X(i) / X(i+1), 1 \leq i \leq n$.

3. If we take $a=b=1$ in (22), we obtain the known result [10, p.349, eq. $(9.7 .8)]$.

\section{References}

[1] A. Erdelyi et. al., Table of Integral Transforms, Vol. I, McGraw-Hill, New York, Toronto and London, 1954.

[2] C. Fox, Some applications of Mellin transforms to the theory of bivariate statistical distribution, Proc. Cambridge Philos. Soc., 53(1957), 620-628.

[3] E. J. Gumble, Statistics of Extremes, Columbia University Press, New York, London, 1960.

[4] E. J. Gumble and L. H. Herbach, The exact distribution of the extremal quotient, Annals of Mathematical Statistics, 22(1951), 418-126.

[5] H. A. David, Order Statistics, John Wiley \& Sons, New York, 1970.

[6] H. Malik and R. Trudel, Distribution of product and quotient of order statistics, University of Guelph Statistical Series, 1975-30(1976), 1-25.

[7] H. M. Srivastava, K. C. Gupta and S. P. Goyal, The H-function of One and Two Variables with Applications, South Asian Publishers, New Delhi and Madras, 1982.

[8] J. D. Gibbons, Non Parametric Statistical Inference, Mc Graw Hill, Koyakusha, Ltd., Tokyo, 1971.

[9] K. Subrahmanian, On some applications of Mellin transform to statistics : Dependent random variables, SIAM Journal of Applied Mathematics, 19(1970), 658-662.

[10] M. D. Springer, The Algebra of Random Variables, John Wiley \& Sons, New York, 1979.

[11] P. Kumaraswamy, A generalized probability density function for double-bounded random process, Journal of Hydrology, 46(1980), 79-88. 